\title{
Protée
}

\section{Performances collectives}

\section{la réception des séries sentimentales par les jeunes téléspectateurs}

\section{Dominique Pasquier}

Volume 30, numéro 1, printemps 2002

Les formes culturelles de la communication

URI : https://id.erudit.org/iderudit/006701ar

DOI : https://doi.org/10.7202/006701ar

Aller au sommaire du numéro

Éditeur(s)

Département des arts et lettres - Université du Québec à Chicoutimi

ISSN

0300-3523 (imprimé)

1708-2307 (numérique)

Découvrir la revue

Citer cet article

Pasquier, D. (2002). Performances collectives : la réception des séries sentimentales par les jeunes téléspectateurs. Protée, 30(1), 67-78.

https://doi.org/10.7202/006701ar
Résumé de l'article

À partir d'une recherche sur les séries sentimentales, cet article interroge les dimensions collectives et performatives de la réception de la télévision par les jeunes téléspectateurs. Le travail de réception est pris dans un faisceau d'interactions sociales qui commandent les modes d'investissement qui sont accordés à une série télévisée. Il est structuré par un travail de présentation de soi : parler de la télévision est une manière, parmi d'autres, de se situer dans un espace socialement normé. Il suppose un travail coopératif pour organiser une expérience commune. 


\section{PERFORMANCES COLLECTIVES: LA RÉCEPTION DES SÉRIES SENTIMENTALES PAR LES JEUNES TÉLÉSPECTATEURS}

DOMINIQUE PASQUIER

La télévision n'est jamais une expérience d'ordre individuel, elle est une expérience qui anticipe et produit des activités collectives. Les premiers travaux sur la réception qui se sont fondés sur l'analyse de la dyade texte/lecteur ont sans doute trop négligé les dimensions sociales et temporelles du processus. La réception est prise dans un faisceau d'interactions qui commandent les modes d'attention et d'investissement qui sont accordés à une émission. Elle est structurée par un travail de présentation de soi et subordonnée à un jeu social de figuration: en parlant de la télévision, on fait apparaître quel type de personne on est et quelle position l'on occupe dans l'espace social. Quelles que soient les stratégies et les rhétoriques utilisées sur la scène sociale, ce travail de mise en scène fait intimement partie de la position spectatorielle elle-même.

Je voudrais interroger ces dimensions performatives de la réception télévisuelle à partir d'un cas précis: celui des séries pour adolescents. Elles ont constitué en France, comme ailleurs probablement, un terrain privilégié pour observer les dimensions collectives de la réception télévisuelle.

Tout d'abord parce qu'elles ont un objet commun: le soir après l'école, c'est la vie avec les camarades d'école qui se poursuit. Mais cette expérience spectatorielle commune n'est pas nécessairement mise en scène de la même façon. On peut avoir vu la même chose tout en se définissant comme des publics tout à fait différents. La même série peut donner naissance à des communautés sociales très dissemblables, même si elles se définissent par un objet commun qui les constitue en public. Les travaux de Roger Chartier sur l'histoire des pratiques de lecture ont d'ailleurs bien montré que le même phénomène existait pour le livre: l'œuvre a la capacité de créer plusieurs sortes de publics, dont aucun ne saurait se réduire à un social "prédécoupé».

Les séries pour adolescents constituent aussi une sorte de répertoire de situations et de caractères, qui permettent d'établir des relations avec la vie sociale. La fiction télévisuelle joue un rôle important dans la diffusion de normes esthétiques et morales; elle propose des modèles relationnels, suggère des scénarios de vie, remanie les repères d'expérience. Les séries alimentent des cultures, à travers le jeu de rôle chez les plus jeunes, les imitations vestimentaires 


\section{HÉLĖNE ET LES GARÇONS}

Cette série d'un producteur français a été diffusée de 1991 à 1994 sur TF1, la plus importante chaîne française de télévision privée. Tournée dans cinq décors intérieurs, elle met en scène les aventures sentimentales de huit étudiants, quatre garçons et quatre filles, dont la vie de couple est sans cesse mise en péril par diverses tentations amoureuses. La série est traitée - et jouée - sur un mode volontairement artificiel, comme un huis clos. On n'y trouve aucune référence à la réalité sociale : les héros sont étudiants mais ils ne travaillent jamais, ils n'ont aucun contact avec le monde extérieur, au soi-disant campus où ils habitent, ils sont coupés de tout lien familial. Toutes les intrigues sont centrées sur la grammaire des relations amicales et amoureuses. Il y a très peu d'action, c'est la circulation de l'information qui constitue l'élément dramatique central : les réactions des personnages, les manières de discuter d'un problème, la mise en œuvre des solidarités.

En France, la série, qui a battu des records d'audience, y compris chez les enfants de moins de 10 ans, a suscité un vif débat dans la presse. On lui a reproché sa pauvreté esthétique et son peu d'ancrage dans la réalité sociale, mais aussi le fait de donner une vision très traditionnelle des rôles sexués. Ensuite, elle a été rediffusée, mais sans susciter un écho comparable à celui qui a accompagné sa première diffusion. 
chez les plus grands, des cultures qui supposent des collectifs et exigent une confrontation aux autres.

Moyen d'apprendre sur les autres, elles sont aussi un moyen privilégié de parler de soi. La déclaration des choix et des préférences est un message personnel destiné à autrui, sous couvert d'un personnage. La télévision agit comme un opérateur d'identification collective et de configuration de la réalité sociale, car elle est un support d'engagement sur la scène sociale. Les processus interprétatifs produisent des normes, non pas au sens de contraintes rigides et non négociables, mais au sens de cadres et de ressources qui permettent de guider et de réfléchir des activités.

Cette recherche sur la réception des séries par les jeunes téléspectateurs s'est fondée sur plusieurs types de matériaux.

- J'ai d'abord soumis un questionnaire à 700 collégiens et lycéens de la région parisienne. Le questionnaire portait sur l'ensemble des séries pour adolescents diffusées à la télévision française et visait à analyser les communautés de goût selon l'âge, le sexe et l'origine sociale - les trois variables de l'échantillon. - Dans le même temps, j'ai réalisé des interviews avec des enfants et leurs parents sur quelques épisodes de la série sentimentale intitulée Hélène et les garçons; série qui a connu un énorme succès d'audience entre 1991 et 1994 auprès des jeunes téléspectateurs, mais aussi des jeunes adultes. Des entretiens avec des étudiants parisiens ont permis de compléter cette partie qualitative de l'enquête.

- Enfin, après de longues démarches, j'ai réussi à obtenir des producteurs de la série Hélène et les garçons l'accès au courrier envoyé par les fans aux comédiens (il arrivait entre 300 à 1000 lettres par jour). J'ai sélectionné environ 7000 lettres en fonction des grandes thématiques qui se dégageaient de l'ensemble: la relation aux comédiens, la compréhension du processus de production, la discussion du thème du couple, ce qui était dit des échanges à propos de la série, que ce soit dans un cadre familial ou dans la sociabilité des pairs.

Ces trois terrains, qui offrent chacun une perspective particulière, furent accompagnés d'un suivi attentif du processus de production (observations de tournages, interviews des producteurs, des scénaristes et des comédiens de la série) et du déroulement d'une tournée de concerts en province de l'interprète principale de la série, Hélène Rollès.

En réalité, il m'apparaît, rétrospectivement, qu'aucune analyse de la réception ne peut se fonder sur un seul matériau d'enquête, et certainement pas sur ce que les premiers chercheurs anglais ont, sans doute abusivement, défini comme une «ethnographie de la réception». Cette dernière méthode, que j'ai cherché à appliquer en début d'enquête, a de très nombreuses limites et entraîne des biais évidents. Par comparaison, le courrier des fans, même s'il ne permet de saisir qu'une posture bien particulière - celle d'enfants très investis dans la série -, constitue un matériau (sur la réception de la télévision) beaucoup plus riche et certainement plus original, non seulement du fait de l'intérêt des lettres, mais surtout parce qu'il n'a pas été produit en réponse à un dispositif d'enquête. Enfin, les réponses au questionnaire, si elles sont traitées pour ce qu'elles sont, à savoir une présentation sociale de soi comme téléspectateur et non une déclaration exacte des choix télévisuels, m'ont permis de comprendre que les variables de sexe, d'âge et d'origine sociale agissaient très directement pour baliser les positions spectatorielles susceptibles d'occuper sur la scène sociale.

\section{LA MISE EN SCĖNE DES IDENTITÉS SEXUÉES}

Il ressort de ces différents matériaux d'enquête que les enfants, même jeunes, sont bien conscients que ce qu'ils disent de la télévision, serait-ce même pour déclarer qu'ils n'aiment pas ou ne regardent pas une émission, engage toute leur personne socialement. Ils apprennent vite à opérer le travail de figuration nécessaire pour entrer en conformité avec les normes et les valeurs des groupes dans lesquels ils cherchent à s'insérer. Ils apprennent à nier certains goûts, à refouler des préférences, ou au contraire à regarder pour entrer dans une communauté de téléspectateurs. Comment s'effectue concrètement ce travail performatif? La mise en scène des identités sexuées 
constitue un exemple particulièrement intéressant. Les liens émotionnels avec la fiction sont soigneusement occultés par les garçons. Ils apprennent vite que, dans la société de leurs pairs, l'identification à un personnage et la manifestation des affects sont des processus à forte connotation féminine. Ils apprennent aussi que les séries qui traitent des sentiments, et surtout bien sûr du sentiment amoureux, sont les plus dangereuses pour eux, car elles sont fortement investies dans la sociabilité féminine. Cette constante se retrouve dans les différents matériaux que j'ai recueillis.

Les observations dans les familles montrent que toutes les scènes renvoyant à la séduction physique féminine ou à l'acte amoureux suscitent immédiatement une réaction de dénégation chez les garçons. Devant un couple qui s'embrasse, un garçon de 6 ou 7 ans est fasciné, mais le problème est que d'autres (dont un chercheur) le voient en train de regarder une scène de ce type; ce qui le conduit à témoigner haut et fort de son désaccord avec ce qui se passe à l'écran: «Encore!/ Et ça recommence!/ Et hop! Encore un baiser! / Ca y est ils vont encore faire l'amour! / Mais ils n'arrêtent pas...». En même temps, il ne quittera jamais une scène de baiser alors qu'il le fera pour d'autres types de scènes (notamment les discussions entre filles dont il peut visiblement se passer). De même, lorsqu'une actrice porte dans un épisode une jupe courte ou une robe décolletée, il va s'exclamer: "Ce qu'elle est moche!». Bref, pour un garçon, cette série serait sans doute agréable à regarder si cela pouvait se faire sans témoin, en se gardant bien par la suite de dire qu'il l'a regardée. Les filles, à l'inverse, surtout quand elles sont jeunes, ne refoulent absolument pas le plaisir émotionnel qu'elles éprouvent (larmes, exclamations, commentaires sur la beauté des personnages). D'ailleurs le courrier des fans (plus de 90\% des lettres sont écrites par des filles) abonde en descriptions très précises sur les modalités physiques de ce plaisir qu'elles éprouvent au voyage fictionnel:

Quand je te voisà la télévision, je ne bouge plus / Ta voix me donne des frissons partout, tu me fais ressentir desémotions/ Quand je te voisj'ai mon cœeur qui bat trop bas...
Les scènes de baisers, particulièrement fréquentes dans Hélène et les garçons, déclenchent ainsi des réactions diamétralement opposées chez les garçons et les filles, comme le montrent ces quelques extraits des réponses à une rédaction proposée par une institutrice ${ }^{1}$, dans un établissement de la banlieue nord de Paris, dont la question était la suivante: «Regardez-vous Hélène et les garçons? Dites en quelques lignes pourquoi vous aimez ou vous détestez ce feuilleton».

Tout d'abord, on constate qu'aucun élève n'a répondu en disant qu'il ne connaissait pas la série et ne l'avait jamais regardée. Sur une classe de trente élèves, tous avaient déjà vu la série, y compris et surtout ce garçon qui écrit: " Je déteste! Même sans regarder, je déteste. Les faux rires ou bien les chansons vraiment nulles à en mourir! Et puis c'est tout le temps pareil».

On constate aussi que les positions des garçons, systématiquement critiques, puisent toutes au même fonds argumentaire: Hélène est une série qui traite de l'amour, c'est donc une série pour les filles. On a une idée des réticences (et de l'ambivalence des déclarations de dénégation!) à travers ces quelques extraits, tous issus de rédactions rédigées par des élèves masculins.

C'est de la crotte puisque c'est pour les filles/Je n'aime pas les feuilletons d'amoureux/C'est pour les filles etça parle d'amour $/ J$ e déteste ce feuilleton carc'est trop sexuel, moije regarde Code Quantum. Les acteurs sont trop sur l'amour, c'est trop sexuel. Ma sœur et ma mère le regardent, c'est pour les filles/Je n'aime pas Hélène et les garçons parce qu'ils se font des bisous dans la bouche toutes les dix secondes / C,a montre des mauvais exemples pour plus tard. Je n'aime pas parce qu' ils s'embrassent /Je déteste parce que toutes les cinq minutes il ya un baiser et je trouve ça dégoûtant/Je n'aime pas Hélène et les garçons et je ne les regarde pas car, vu leurs extraits de feuilleton, ils ne fontques'embrasser et ils se prennent pour les centres du monde. Il faut que ça cesse! Ils me gâchent le plaisir de regarder.

Certaines élèves féminines de la même classe ont elles aussi dénoncé les baisers dans la série. Mais elles l'ont fait de manière très différente, en se plaignant plutôt du caractère répétitif de ces scènes ou du 
manque de naturel des comédiens en train de s'embrasser. Bref, les baisers peuvent aussi agacer les filles, mais c'est en tant qu'artifice narratif et non en tant que symbole de la vie sexuelle.

Dans le questionnaire soumis aux collégiens et lycéens, les réponses à la question sur leur personnage préféré montrent que les jeunes interrogés manient parfaitement bien la rhétorique de mise à distance et de présentation de soi à travers leurs discours sur la télévision. Il y a de profondes différences entre les réponses des filles et celles des garçons: non seulement les uns et les autres ne choisissent pas les mêmes personnages, mais ils ont aussi une argumentation pour justifier leurs choix qui repose sur des registres opposés.

Les choix et les argumentations des répondants masculins présentent trois grandes caractéristiques. Tout d'abord, leur personnage préféré est presque toujours un personnage masculin qui n'a pas de vie de couple régulière ${ }^{2}$. Seuls les plus jeunes des répondants dérogent à cette règle. Ce personnage sera aussi choisi dans des séries qui traitent de la vie sentimentale de façon accessoire. Ces personnages ne sont pas aimés parce qu'ils sont beaux mais parce qu'ils sont transgressifs. C'est là la deuxième caractéristique des réponses masculines: un personnage n'est jamais choisi au nom de considérations physiques. On l'aime pour ce qu'il fait et non pour ce qu'il est. Les héros préférés des garçons sont des bons vivants, malins plus qu'intelligents, rebelles à l'ordre scolaire et à l'autorité. Ce qui plaît chez eux, c'est leur capacité à se sortir de situations qui ne sont pas à leur avantage. Ce ne sont pas des personnages de gagnants et pourtant leur audace finit toujours par payer. C'est la revanche de la débrouillardise sur l'héritage social. Des personnages très proches finalement des héros de la culture populaire, tels que les décrit Richard Hoggart (1970).

Ce que les répondants disent apprécier par-dessus tout chez leur personnage préféré, c'est l'humour. Cette qualité, qui apparaît peu dans les critères évoqués par les filles, vient en tête de leur argumentation. Si les garçons disent aimer les personnages qui les font rire par leur désinvolture, c'est aussi que ces derniers risquent moins que d'autres de les faire passer pour des téléspectateurs trop investis dans une fiction. Le rire est une émotion moins compromettante et il est socialement possible pour un garçon de s'intéresser à des histoires d'amour si elles sont placées sous le signe de l'humour et non celui des sentiments.

Les réponses des filles su sujet de leur personnage préféré sont très différentes. On constate déjà qu'elles n'hésitent pas à choisir un personnage masculin (c'est le cas de $54 \%$ d'entre elles) alors que les garçons se gardaient bien de choisir un personnage féminin ${ }^{3}$. Les $11 \%$ de garçons qui l'ont fait (11\%) ont presque tous entre 10 et 12 ans: ils ne savent pas encore que la préférence pour un personnage féminin est perçue par les autres garçons comme un choix socialement dangereux. Ce choix peut en effet signifier qu'une part de séduction entre dans leur relation à la fiction. Car les filles ne cachent pas que le choix d'un personnage est motivé par des considérations physiques: un personnage plaît parce qu'il est séduisant. La chose vaut également pour les personnages féminins, qui sont choisis par 33\% des répondantes. L'apparence, le charme, la beauté, les vêtements, voilà donc ce qui motive en premier lieu les choix féminins. Toutefois un personnage n'est jamais aimé uniquement pour son physique: la gentillesse et le dévouement font la différence entre les personnages beaux et généreux (qui sont les personnages préférés) et les personnages beaux mais durs ou égoïstes (qui sont les personnages les moins aimés ${ }^{4}$ ).

On constate aussi qu'en avançant en âge, les répondantes s'intéressent de plus en plus à des personnages qui ont des problèmes et elles ont, de manière générale, tendance à se rapprocher des positions masculines: elles continuent à regarder les séries sentimentales mais en manifestant plus de distance critique: elles dénoncent les intrigues qui se répètent, les dénouements toujours heureux, les histoires dont on peut prévoir le déroulement à l'avance. Elles se mettent aussi à préférer les personnages révoltés aux personnages doux. En revanche, à aucun moment, elles ne renoncent à déclarer être attirées physiquement par certains 
personnages masculins ou admirer la beauté d'un personnage féminin. Afficher un lien fort avec une fiction ou une relation forte avec un personnage n'est donc pas vécu par une fille comme un facteur de fragilisation de l'identité. Toutefois, ce qui devient menaçant pour elles, ce sont les séries ou encore les personnages trop simplistes, ceux que les petits préfèrent, et vis-à-vis desquels les adolescentes plus âgées doivent prendre des distances, sous peine d'être ridiculisées par leur groupe. Il y a des choix qui s'opèrent alors, qui conduisent à préférer les séries américaines aux séries françaises - comme les garçons le font dès le départ (les résultats du questionnaire montrent, comme d'autres enquêtes, qu'il est accordé aux séries étrangères une certaine latitude dans le traitement de la réalité sociale, ce qui n'est pas le cas dans les séries françaises ${ }^{5}$ ).

\section{DES PUBLICS AUX COMMUNAUTÉS SOCIALES}

Toute expérience sociale de la télévision suppose aussi un travail coopératif pour organiser une expérience commune. Un public est public par rapport à d'autres publics, face auxquels il cherche à se définir et à se manifester. Les différents matériaux que j'ai pu recueillir permettent d'analyser concrètement le travail d'ajustement qui est opéré pour que le «moi» du téléspectateur se transforme en un «nous», un être ensemble face à la télévision. Chaque communauté a ses règles, ses codes, ses modes d'engagement, ses mises en scène de la distance. Chaque communauté repose aussi sur le sentiment d'appartenir à un collectif, dont on peut tirer d'importants profits de sociabilité. On peut en donner quelques exemples à partir de l'enquête sur Hélène et les garçons.

\section{La double communauté des fans}

Première communauté, la communauté des fans. C'est bien sûr la communauté qui se met socialement en scène de la manière la plus visible. La fan est quelqu'une qui s'affiche comme fan. Tout d'abord visà-vis de son idole: le début de la plupart des lettres est consacré aux preuves d'amour que la correspondante donne à Hélène. Des listes de tous ordres - les noms de tous les comédiens de la série, les titres de toutes les chansons d'Hélène, toutes les paroles d'une des chansons -, des montages de photos et d'images tirées de la série ("... la preuve est derrière", écrit Amadine qui a collé au verso de sa lettre dix-sept autocollants d'Hélène). La fan porte aussi ostensiblement les couleurs de celle qu'elle aime ("Je me suis acheté une tenue de toi où tu es en photo sur le pull, cela te fait de la publicité»). Il suffit d'entrer dans les chambres des fans pour le comprendre: couvertes de posters du sol au plafond, on entre dans un univers absolu, exclusif:

J'ai 120 posters de toi et des autres d'Hélène, même que ma mère m'a obligée à enlever ceux qui étaientsur ma porte, quel dommage! Ma mère ditqu'on m'a mis du papier peint pour rien! Sache que je suis l'une de tes plus grandes fans. Ma chambre toutle monde la surnomme "Lacaverne d'Hélène» et c'est vrai, il n'y a que des photos de toi. (Émilie)

Du soir au matin, la fan vit avec Hélène: elle dort dans des draps "Hélène", s'habille et se coiffe comme Hélène, fait son travail scolaire dans des cahiers «Hélène», joue à Hélène et les garçons pendant les récréations, regarde la série en rentrant, avant d'écouter des chansons d'Hélène, seule dans sa chambre en pleurant d'émotion et en parlant avec ses posters.

Mais surtout la fan est quelqu'une qui est entourée. L'intégration dans un réseau d'échange et de discussion est indispensable. Isolée, une fan serait condamnée à redevenir une simple téléspectatrice, éventuellement plus assidue que d'autres. La fan, elle, fait beaucoup plus. Elle participe à des jeux de rôles, elle répercute les informations qu'elle a lues dans l'espoir d'en apprendre d'autres en retour, elle échange des images en double pour finir son album "Panini», elle se tient informée des derniers objets qui sont mis sur le marché. Pour tout cela, il faut des partenaires. Ces partenaires, elle les trouve à l'école bien sûr, mais aussi dans le voisinage ou sur les lieux de vacances. Les lettres écrites pendant le mois d'août 1994 montrent bien que, même en camping, sans possibilité de suivre les épisodes télévisuels, les liens 
avec la série peuvent quand même exister grâce aux échanges avec d'autres enfants.

Cette dimension collective existe dès le départ. On ne devient pas fan toute seule devant son poste. Il faut des intermédiaires, généralement une autre fan dans l'entourage proche, qui va s'employer à convertir la nouvelle initiée en lui révélant des informations sur la vraie vie des personnages.

Chère Hélène, je m'appelle Linda etj'ai 11 ans. Je ne t'aimais pas beaucoup avantqu'une de mes copines m'ait aidée à mieux te connaitre et grâce à elle maintenantje t'aime beaucoup. C'est Stéphanie qui m'a aidée à mieux te connaitre et elle est très sympathique, tu verras car elle aussi t’a écrit.

Sur ce point, les témoignages issus du courrier sont tout à fait concordants: on devient fan en parlant d'Hélène avec d'autres enfants qui l'aiment déjà et non en regardant la télévision ("J'ai une copine qui est fan de toi, c'est elle qui m'a donné des détails sur toi. Avant je te connaissais que par la télévision»). Le processus s'inscrit dans une dynamique collective. Ou plutôt dans une succession de collectifs qui s'emboîtent les uns dans les autres, comme des poupées russes.

Premier collectif: la meilleure amie. Elle peut avoir acquis ce statut de meilleure amie parce qu'elle était fan elle aussi ou après avoir été convertie. En tout cas, ces tandems sont très soudés et il y est fréquemment fait allusion dans le courrier. Avec la meilleure amie, on partage. On partage des objets ( "Ma meilleure amie Florence m’a prêté le livre Je m'appelle Hélène et la cassette vidéo, et moi je lui ai prêté le disque laser et le $C D$ deux titres»). On partage des informations ( "Tous les jours je regarde Hélène et les garçons à 18h25, c'est vraiment bien, quelquefois je le loupe car je suis de sortie, alors je demande à ma copine Amandine ce qui s'est passé»).

Deuxième forme de collectif: le jeu. Un peu plus large, en général quatre à cinq amies (comme le nombre des personnages féminins de la série), ce collectif fonctionne surtout autour de jeux de rôles. Ils sont féminins dans le cas d'Hélène et les garçons ( «Les garçons on se les imagine», écrit Belinda), mais, pendant ce temps, d'autres jeux de rôles, cette fois masculins, se déroulent autour des «mangas» japonais ou des dessins animés de superhéros.

Entre fans, il n'y a toutefois pas que de la coopération, il peut y avoir de la concurrence. C'est le cas des enfants qui sont fans elles aussi, mais qui ne font pas partie du même groupe de jeu, ce qui arrive souvent dans les cours d'école. Il s'engage alors une véritable compétition. L'enjeu? Obtenir d'Hélène quelque chose que les autres n'ont pas. En d'autres termes, avoir la preuve d'un lien privilégié. Les lettres se font insistantes et l'on sent qu'il y a derrière ces demandes bien des rivalités. Anne Sophie demande des photos dédicacées de tous les acteurs de la série (dont elle a recopié patiemment les noms de famille), y compris des seconds rôles que l'on n'aperçoit que rarement: «Merci de m'envoyer tout le plus vite possible car je fais un concours avec les copines de mon école pour savoir qui en aura le plus".

Il y a donc des alliées et des concurrentes. Il y a aussi des ennemis. Au sein des écoles primaires, les détracteurs d'Hélène sont toujours des garçons, et toujours des garçons qui se moquent de la série parce qu'elle parle d'amour. Les garçons sont culturellement ennemis des fans d'Hélène, qu'ils aiment ou non la série, qu'ils la regardent ou pas. C'est une position de principe et les filles le comprennent bien ainsi: «Mon frère se moque tout le temps de moi quand je parle de toi, mais il faut le comprendre c'est un garçon", écrit Audrey, 10 ans.

Avec l'entrée au collège, le nombre et la pression des opposants s'accroissent. Hélène n'est plus seulement considérée comme une série pour les filles, mais aussi comme une série pour les petits. Les fans sont donc confrontées à un nouvel obstacle: si elles continuent à dire qu'elles aiment la série, elles sont cataloguées comme puériles dans leur entourage scolaire, non seulement par les garçons, mais cette fois aussi par des filles. Symptomatiquement, toutes les lettres faisant état de ce problème sont écrites par des filles ayant entre 12 et 15 ans: elles ont quitté le primaire où il était permis d'aimer Hélène sans passer pour infantiles; elles ne sont pas encore entrées au lycée où il leur sera impossible de se déclarer fan si ce 
n'est sur le mode parodique. «Mes copines me prennent pour une gamine parce que je vous aime», écrit Myriam, 14 ans.

Il ya dans ma cour des garçons et des filles qui disent que votre série c'est pour les gamins, Moi je leur dit non, car mes parents la regardent, ma tante la regarde et dans cette série on nous montre ce qu'il ne faut pas faire comme la drogue, se disputer. Alors je défends votre série car je ne voudrais pas que ça s'arrête.

(Mélanie, 14 ans)

Il y a les fans de l'entourage avec qui l'on discute et l'on joue, mais il y a aussi les autres fans, celles qu'on ne connaît pas directement, mais dont on connaît l'existence par la presse ou des émissions de radio et de télévision. Toutes les petites fans savent ainsi qu'Hélène a reçu des milliers de lettres, qu'elle ne peut sortir dans la rue sans être suivie par des dizaines d'enfants qui veulent un autographe, et que ses concerts font salle pleine. Bref, elles savent qu'elles ne sont pas uniques. À distance, d'autres fans collectionnent les mêmes objets, prononcent les mêmes mots, jouent aux mêmes jeux. «Nous sommes deux à t'écrire mais beaucoup à t'aimer», disent Amandine et Sabrina dans leur lettre à Hélène.

Microcommunautés, macrocommunauté. On retrouve là le mécanisme de communautés en deux temps étudié par Daniel Dayan et Elihu Katz. Comme pour les grandes cérémonies télévisées, la série constitue «une expérience collective à domicile», à travers une «multitude de microcélébrations orientées au même moment vers le même centre symbolique» et qui «font du foyer une expérience sociale» (1996, p. 146). En réalité, la participation à la communauté imaginée des autres fans est tout aussi importante que l'appartenance à ces communautés humaines réelles que l'on vient d'évoquer. C'est le nombre de ses admiratrices qui consacre Hélène dans sa supériorité. Et qui, du même coup, conforte les fans dans le sentiment d'avoir fait le bon choix. Alberoni (1994) dirait que ces fans épousent le désir de la multitude. Il y a quelque chose de rassurant à faire corps ainsi, et lorsque la série est attaquée par la presse ou maltraitée par les responsables de la télévision (un changement d'horaire par exemple), les fans écrivent au nom de l'immensité de leur groupe. Elles sont là pour la défendre, et plus nombreuses elles sont, mieux c'est:

Je t'ai écritcette lettre pourque tu saches que j'existe et que je suis une de tes fans parmi beaucoup d'autres filles de mon âge. Tu ne me connais pas mais tu vas avoir une fan de plus.

(Agnès, 10 ans)

En même temps, le nombre inquiète. Des milliers, des millions, les fans hésitent ( CCombien as-tu en gros de fans?", demande Floriane). Tout d'abord, cette masse risque de noyer les individualités et d'empêcher Hélène de traiter chaque correspondante comme elle le souhaite, c'est-à-dire comme une personne unique en son genre et digne d'un intérêt particulier. Anaïs parle de sa lettre comme d'une aiguille dans une meule de foin et Aurélie comme d'une goutte d'eau face à l'océan de lettres qu'Hélène reçoit, toutes deux exprimant chacune à sa manière ce que de très nombreuses fans disent redouter: être perdues dans la masse.

En réalité, quels que soient le ton et les attentes, les fans ont toujours conscience de faire partie d'un groupe dont elles ne connaissent pas les membres et qui pourtant est présent, obstacle ou soutien, dans leur relation à l'idole.

\section{Communautés critiques et parodiques}

Le public d'Hélène et les garçons n'est pas, tant s'en faut, uniquement constitué de jeunes fans. C'est même un des paradoxes les plus intéressants de la série: elle a drainé chez les adolescents tout un public très critique, mais néanmoins fidèle devant son poste. En quelque sorte des téléspectateurs malgré eux. On a là un cas de figure très différent de celui des jeunes garçons du primaire qui refusent de regarder - ou de dire qu'ils regardent - de peur de compromettre leur identité masculine. Ou de celui des collégiennes qui découvrent que la série risque de les faire passer pour infantiles auprès de leur entourage. Ces téléspectateurs critiques ne se cachent pas: ils avouent regarder Hélène et les garçons, mais cet aveu se fait au prix de tout un travail rhétorique visant à montrer que leur manière de regarder est bien différente de celle du public des 
petites fans: ils regardent la série en la trouvant insipide et débile. C'est même pour toutes ces raisons qu'ils ne veulent pas rater un épisode.

En réalité, leur position est moins paradoxale qu'il n'y paraît. Une telle rhétorique a son utilité. Tout d'abord, elle permet de regarder la série: en affichant une position très critique, ces adolescents marquent bien les différences entre eux et le public soi-disant mystifié des enfants du primaire. Ils regardent la même chose, certes, mais avec un regard qui est diamétralement opposé. Ensuite, c'est une manière de se solidariser avec d'autres, qui portent le même regard critique: une sorte de NOUS qui se constitue contre le IL de l'écran, comme l'analysait Tamar Liebes à propos des téléspectateurs de Dallas qui se disent fascinés par le personnage de JR tout en le détestant (Liebes, 1994) ${ }^{6}$. On a là un collectif qui se structure autour des mêmes rejets: ensemble on se moque du personnage d'Hélène, ensemble on trouve ridicule la bande-son de rires préenregistrés, ensemble on fait des plaisanteries sur les histoires à répétition dans la cafétéria. La position de téléspectateur critique n'est viable qu'au sein d'une communauté. Ces extraits d'un article, rédigé dans un journal lycéen par une élève de seconde de la banlieue ouest, donnent le ton:

Ce mois-cij'ai choisi de vous parler des séries télé qui nous envahissent peu à peu et dont on peut estimer le niveau intellectuel à environ zéro. Ces petites séries qui regorgent d'histoires de cour et de mecs et de nanas au physique décourageant, sans oublier les inévitables rires enregistrés, font notre bonheurà tous. Mais bien sûr vous ne regardez jamais de telles c... (ça fait ringard devant les copains!). Menteurs! On vous connaît, dès que vous arrivez chez vous, vous vous vautrez dans le canapé et vous vous branchez sur «Hélène et compagnie»... Mais n'ayez crainte, votre réputation estsauve: vous n'êtes pas tout seul!...

Interrogés sur Hélène et les garçons, une partie des étudiants tiennent des propos similaires. "C'est une idiotie de plus, mais qui prend moins la tête que les autres. C'est un bon sujet de conversation!", explique un étudiant de 22 ans.
Tout le monde déteste mais tout le monde regarde, surtout quand on fait des baby sitting, dit cette étudiante de 19 ans, c'est commeCasimir, ça crée des liens subcultures. Mais Casimir, c'était mieux! La seule excuse valable pour regarderc'estqu'en rentrant des cours on estcrevés. On se laisse porter par le flot, on ne réfléchit pas. C'est parfait pendant les révisions d'examens, c'est tellement niais. / On en parle beaucoup mais au 3e ou 4 e degrépour se moqueret ridiculiser les personnages, quelquefoison s'amuseà faire des rires comme dans le feuilleton, peutêtre aussi pour détournerdu faitqu'on regarde quand même...

Au-delà de la critique, la parodie. La communauté parodique recrute ses membres chez les étudiants des universités les plus sélectives ou des grandes écoles, et compte bon nombre d'étudiants masculins. Elle concerne donc des téléspectateurs suffisamment âgés, dotés d'une culture plus vaste pour mieux se distinguer du public des petites fans du primaire. Et c'est bien parce qu'ils sont différents qu'ils peuvent entrer dans le jeu parodique.

Ce qui est parodié n'est pas la série elle-même mais ses fans. Ou plutôt l'idée qu'on se fait des fans d'Hélène dans les milieux étudiants: des fans naives et crédules, qui prendraient les personnages et les histoires au pied de la lettre. En ce sens, la communauté parodique est profondément dépendante de l'existence attestée d'une communauté de fans véritables, qui soit suffisamment nombreuse et visible pour que la parodie «fasse sens" comme pratique distinctive. Prenons-en pour preuve le fait que les lectures parodiques ont cessé d'exister dès que la dynamique sociale autour d' Hélène et les garçons est devenue moins importante et que l'audience de la série s'est mise à décliner.

Ces étudiants ne cherchent pas à se moquer des contenus de la série comme le font les membres des communautés critiques. Ils ne cherchent pas non plus à la dénigrer. Ils jouent à endosser, le temps du jeu, la personnalité de celles qui sont supposées regarder la série avec les yeux de l'adoration sans réserve et de l'identification sans retenue. Les faux-fans se comportent le plus possible comme les vraies fans: ils se font raconter par des amies les épisodes qu'ils ont manqués ("On s'arrange pour qu'il y en ait toujours une 
qui puisse regarder", explique Séverine qui suit la série avec deux autres amies de la même classe de droit), ils discutent des intrigues, ils cherchent à avoir des informations inédites sur les comédiens pour pouvoir les raconter aux autres faux-fans (on m'a aussi souvent demandé, dans les interviews d'étudiants ou dans les familles avec des petites fans, si j'avais rencontré Hélène et comment elle était!), ils disent trouver la série exceptionnellement réussie. On retrouve là le même principe de double communauté. Un petit groupe de (faux) admirateurs local, au sein duquel se font les échanges d'informations et de discussions, et une communauté imaginée constituée des autres étudiants dont on sait pertinemment qu'ils jouent à la même chose, sans doute en même temps (les intercours, le Resto U). Toutefois, les faux-fans ne vont pas jusqu'à écrire à Hélène ou collectionner des objets (bien qu'on m'ait à plusieurs reprises demandé d'obtenir une photo dédicacée "pour accrocher au-dessus de mon lit» et qu'une étudiante ait offert un album «Panini Hélène» à l'une de ses amies pour son anniversaire - cette dernière ne l'a en revanche jamais rempli car «elle n'a pas eu le temps d'acheter les images»). Ils n'envisageraient pas non plus une seconde d'assister à un des concerts d'Hélène. Il n'y a donc aucun contact physique avec les vraies fans (une étudiante a confié avoir regardé des épisodes avec sa cousine de 7 ans chez leur grand-mère l'été et s'être sentie gênée, car «ça ne faisait pas pareil que de regarder après la fac") et surtout pas de désir d'entrer en contact avec Hélène. C'est une grande différence. Enfin, il y a toujours un moment, dans les interviews, où la vérité est dite ("non il faut quand même reconnaître que c'est supérieurement débile»), moins comme une confidence, mais plutôt pour s'assurer que l'interviewer ne s'est pas mépris sur les positions réelles de son interlocuteur. Ces positions ne sont d'ailleurs pas si claires: certes les étudiants jouent à aimer Hélène et en ce sens ce ne sont pas de vrais fans. Mais, en même temps, ils regardent assidûment la série et passent un temps non négligeable à en parler, sans qu'on puisse être sûr que le plaisir qu'ils en tirent soit uniquement au second degré. Ils le confessent eux-mêmes, au bout de quelques épisodes ils sont pris par le jeu et détestent sincèrement en rater un.

J'arrive chez moi, je mets Hélène et les garçons, c'est la détente totale, je me vide la tête après les cours, j’adore. Je me marre d'avance en pensantà ce qu'on va se raconter le lendemain.

À l'Institut d'Études politiques de Paris, un étudiant de dernière année a même fondé une association d'élèves qui a mené sa campagne de 1993, pour l'élection au Bureau des Élèves, sur le thème "Hélène et les garçons c'est génial». «L'idée m’est venue en regardant Voisin voisine " ${ }^{7}$, explique le responsable de l'initiative.

On passait des nuits blanches au foyer, à quatre ou cinq dans la chambre à regarder Voisin voisine ou Tendresse et Passion. On était morts de rire. J'ai découvert Hélène ensuite et vraiment ça battait des records. Au début on a juste fait des posters avec des extraits d'Hélène et deVoisin voisine. Puis après on a commencéà diffuser des tracts avec des résumés d'épisodes. Lesgens venaient nous demander: qu'estce qui s'est passéhier? Alors on a commencéa faire des résumés écrits qu’on affichait tous les jours. Ca répondaità une demande. Pendant la période électorale on avait une table avec des exemplaires de Bravo Girl!! et une pétition à signer pour le retour de Johana dans la série. On passait aussi des chansons d'Hélène sur la péniche (le hall des sciencespo), ça marchait très bien, à la fin tout le monde connaissait les paroles, on se marrait. Quand on parle d'Hélène à sciencespo pas besoin de dire que c'est nul, on ne critique pas, il suffit de mettre un poster, tout le monde comprend. On n'a pas besoin d'avoir l'attitude «c'est nul, c'est nul", tout le monde le sait. On était huit sur la liste électorale du GAP, on regardaittous les épisodes en prenant des notes pour faire les résumés. À la fin, on était plusieurs à être vraiment accros, c'était dingue. En tout cas ça a marché, on a eu $9 \%$ des voix grâce à Hélène.

La lecture de la télévision fonctionne donc toujours sur un mode communautaire. Les lectures fans et les lectures parodiques sont les seules à officialiser cette communauté, mais les lectures ordinaires n'y échappent pas, même s'il s'agit de communautés moins institutionnalisées et plus mouvantes en 
fonction de sous-groupes consolidés sur la base d'une classe d'âge, d'une appartenance sexuelle ou d'un milieu social; ces trois variables agissant ensemble la plupart du temps pour redéfinir des sous-groupes localement constitués. Ces collectifs sont interdépendants, même si chacun entretient sa cohérence propre et se fonde sur un accord quant aux principes qui doivent guider la lecture. Les collectifs de lecture se constituent dans un espace social où s'exercent des rapports de force. Tout public est face à d'autres publics, dont il connaît l'existence et par rapport auxquels il a à se justifier. Une grande partie du travail performatif, pour marquer son appartenance à un type de public, s'effectue en fonction de la manière dont les autres publics se mettent en scène socialement.

On remarquera aussi que la lecture soi-disant fan, c'est-à-dire la lecture sans distance qui est attribuée aux fans, est centrale dans le positionnement des lectures les unes par rapport aux autres. C'est une position qui semble n'être en réalité occupée par personne, et pourtant:

- Elle fonde les tentatives des fans d'entrer dans une relation particulière avec l'idole ( «je sais que la plupart de tes fans t'aiment pour ton personnage, moi pas; il ya des fans qui croient que...»);

- Elle fonde les lectures parodiques: parodier c'est jouer au fan pathologique, celui qui ne pourrait pas vivre sans son émission, et qui vit à l'intérieur de celleci comme si elle formait un univers réel;

- Elle fonde même les lectures ordinaires, puisqu'elle est affectée à des sous-catégories (les filles, les petits) dont on va chercher à se démarquer dans la présentation de soi comme téléspectateur sur la scène sociale.

\section{D' HÉLĖNE À LOFT STORY: LA CULTURE CAMP}

Comme le souligne Naomi Klein, la renaissance des opérations de résistance culturelle aux messages publicitaires «a beaucoup à voir avec les technologies nouvellement accessibles qui ont largement facilité la création et la diffusion des parodies publicitaires" (Klein, 2000, p. 340). Mais, rappelle-t-elle aussi, de tels mouvements d'activisme anti-publicité existaient dès les années 1930, et le développement de sites parodiques, sur Internet par exemple, ne permet finalement que de donner plus d'ampleur et d'accessibilité à ces formes d'expressions esthétiques de la dérision. Tout comme certains activistes détournent des logos et des campagnes de publicité, une partie du public de la télévision pratique le «faire semblant». Le bricolage des cultures, si bien étudié par les chercheurs des cultural studies à propos des emprunts et des détournements des objets culturels par les jeunes Anglais issus des classes ouvrières (Hebdidge, 1979), est une forme de relation à la télévision chez les jeunes qui tend certainement à prendre le pas sur la critique dénonciatrice et rationnelle que l'on peut lire dans les colonnes de la presse cultivée. Dire qu'une série de télévision est stupide et ridicule, c'est finalement entretenir un rapport de croyance au média. C'est supposer qu'il doit dire des choses utiles, fondées, intelligentes. Or, c'est là une position qui a de plus en plus de mal à trouver ses points d'appui dans un univers télévisuel, dont les objectifs commerciaux sont avoués, qui est marqué par une énorme croissance de l'offre et par une circulation accrue des programmes hors des frontières nationales. Les grands feuilletons américains des années 1980, comme Dallas ou Dynasty qui ont été l'objet de nombreuses recherches sur la réception, ont permis de comprendre que le jeu et l'ironie (ce que les Anglo-Saxons appellent la lecture camp) étaient des postures spectatorielles qui avaient toute leur logique. Les communautés homosexuelles américaines sont parties de l'outrance de l'esthétique de Dynasty pour en faire une série culte, renvoyant à la stigmatisation sociale du maniérisme homosexuel (Gripsrud, 1995). Les admiratrices des soap operas, étudiées par Mary Ellen Brown (1994), jouent du fait d'être «accros» comme une manière d'affirmer haut et fort que les hommes ont bien raison de mépriser les femmes intellectuellement. Dans ces deux cas, on le voit, l'autodérision (revendiquer le maniérisme, revendiquer le plaisir à regarder des programmes dont on sait qu'ils sont jugés stupides) fonde le sentiment d'appartenir à un groupe qui emploie les mêmes codes 
de lecture. Il n'en a pas été autrement pour les étudiants qui se déclaraient fans d'Hélène et les garçons. Et dix ans plus tard, le phénomène social autour de Loft Storys'est constitué sur les mêmes bases. Comme un jeu qu'il faut prendre apparemment au sérieux pour être sûr de vraiment s'amuser. Internet a simplement permis de monter cela à plus grande échelle. Des dizaines de sites web parodiques, mêlant informations et rumeurs, vraies et fausses images, proposant des lectures décalées ou dérisoires en transposant la série dans d'autres mondes sociaux ou dans d'autres scénarios, offrent à des communautés plus larges un lieu de pratiques collectives de la dérision qui permettent de participer à une sousculture commune dont les gratifications de sociabilité sont évidentes. La déclinaison sur d'autres médias et d'autres supports de formes détournées d'une série télévisée (dans le cas de Loft Story, principalement la radio et les sites web) conduit à une situation paradoxale: la série n'est là que pour alimenter des formes d'échanges culturels qui lui sont extérieures. Et les travaux sur la constitution des publics télévisuels devront désormais intégrer cette nouvelle dimension d'une réception multimodale où le rapport à la culture se joue à plusieurs niveaux et sur plusieurs supports.

\section{Notes}

1. De CM1/CM2, soit des enfants ayant entre 9 et 11 ans (mars 1994). 2. Ce qui leur laisse peu de choix au sein de l'ensemble des séries: les trois personnages qui répondent à cette définition sont Zack de Sauvés par le Gong, Will du Prince de Bel Air et Parker Lewis de la série du même nom.

3. M.J. Chombart de Lauwe, dans un travail sur les personnages des médias préférés par les enfants, constatait déjà cette prééminence des choix de personnages masculins. Mais elle l'attribuait à un problème d'offre: si les enfants préfèrent des personnages masculins, c'est parce que les personnages féminins importants sont très peu nombreux. Cette hypothèse ne peut pas fonctionner à propos des séries pour adolescents qui comptent un grand nombre de personnages féminins principaux.
4. En réponse à la question "Quel est le personnage que tu aimes le moins?".

5. La question posée était «Est-ce que ce que tu vois dans ces séries ressemblent à ce que tu connais autour de toi?». Plusieurs travaux (voir notamment Hjort, 1986) ont permis de mettre en évidence que les attentes de réalisme étaient moins fortes à l'égard des fictions étrangères que des fictions nationales.

6. Voir aussi les travaux de I. Ang (1985) sur les téléspectatrices de Dallas qui avouent l'ambiguïté de leur propre attitude ( Je suis féministe et j'adore Dallas " /Je suis de gauche et $j$ 'adore Dallas ").

7. Une fiction destinée à remplir les obligations de quotas d'œuvres d'expression française qui venaient d'être imposés aux chaînes. Fiction produite avec de tous petits budgets, un seul décor et des comédiens débutants, diffusée sur la 5 toutes les nuits à $1 \mathrm{~h}$ du matin.

8. Revue pour adolescentes qui traite des premières expériences sexuelles.

\section{RÉFÉRENCES BIBLIOGRAPHIQUES}

AlBeroni, F. [1994] : Le Vol nuptial, Paris, Plon. ANG, I. [1985]: Watching Dallas. Soap Opera and the Melodramatic Imagination, Londres, Routledge.

BRown, M. E. [1994]: Soap Opera and Women's Talk, Londres,

Routledge.

Chartier, R. [1996]: Culture écrite et société, Paris, Albin Michel;

[1998]: Au bord de la falaise, l'histoire entre certitudes et inquiétude, Paris Albin Michel.

Chombart de Lauwe, M.-J. et C. Bellan [1979]: Enfants de l'image,

Paris, Payot.

DAYAN, D. et E. KATZ [1996] : La Télévision cérémonielle, Paris, P.U.F.;

[1992]: "Les mystères de la réception ", Le Débat, n 71.

GRIPSRUD, J. [1995]: The Dynasty Years. Hollywood Television and Critical Media Studies, Londres, Routledge,

HEBDIDGE, D. [1979]: Subcultures, the Meaning of Style, Londres,

Routledge.

HjorT, A. [1986]: When Women Watch TV. How Danish Women Perceive the American Series "Dallas" and the Danish Series "Daughters of War", Copenhagen, Danish Broadcasting Corporation, rapport multigraphié. Hoggart, R. [1970]: La Culture du pauvre, Paris Éd. de Minuit, préface de J.-C. Passeron.

KLEIN, N. [2000] : No Logo, Toronto, Alfred Knopf.

LIEBES, T. [1994]: "À propos de la participation du téléspectateur ", Réseaux, n 64, 93-107.

Liebes, T. et E. KATZ [1990]: The Export of Meaning, Cross Cultural Readings of Dallas, New York, Oxford University Press.

PASQUIER, D. [1994a] : "Vingt ans de recherches sur la télévision. Une sociologie post lazarsfeldienne? », Sociologie du Travail, n 36, 1;

[1994b] : "Hélène et les garçons: une éducation sentimentale», Esprit (juin);

[1996]: "Teen Series'Reception: Television, Adolescence and Culture of Feelings ", Childhood, vol. 3, n³, 351-375;

[1999]: La Culture des sentiments. L'Expérience télévisuelle des adolescents, Paris, Éd. de la Maison des Sciences de l'Homme;

[2001]: "Media at Home: Domestic Interactions and Regulation ", dans S. LivingstonE et M. BOVILL (sous la dir.), Children and ther Changing Media Environment, Los Angeles, Erlbaum. 